\title{
Chemical Doping and Etching of Graphene: Tuning the Effects of NO Annealing
}

\author{
${ }^{1}$ G.K. Rolim, ${ }^{2}$ G.V. Soares, ${ }^{2}$ H.I. Boudinov and ${ }^{3}$ C. Radtke*
}

${ }^{1}$ Programa de Pós-Graduação em Microeletrônica, UFRGS, 91509-900 Porto Alegre, Rio Grande do Sul, Brazil

${ }^{2}$ Instituto de Física, UFRGS, 91509-900 Porto Alegre, Rio Grande do Sul, Brazil

${ }^{3}$ Instituto de Química, UFRGS, 91509-900 Porto Alegre, Rio Grande do Sul, Brazil

*Corresponding author: Tel.: +55 5133088189 E-mail: claudio.radtke@ufrgs.br 
SUPPORTING INFORMATION

The $\mathrm{C} 1 \mathrm{~s}$ region of the XPS spectrum of a pristine graphene $/ \mathrm{SiO}_{2} / \mathrm{Si}$ sample is shown in the following, evidencing that no prominent deposition of carbonaceous contaminants is observed. The intensity of the high binding energy shoulder of the signal is diminished following $\mathrm{CO}_{2}$ and NO annealings, observation related to the cleaning effect of these annealings. The low binding energy component in the $\mathrm{CO}_{2}$ annealed sample corresponds to products of PMMA degradation.

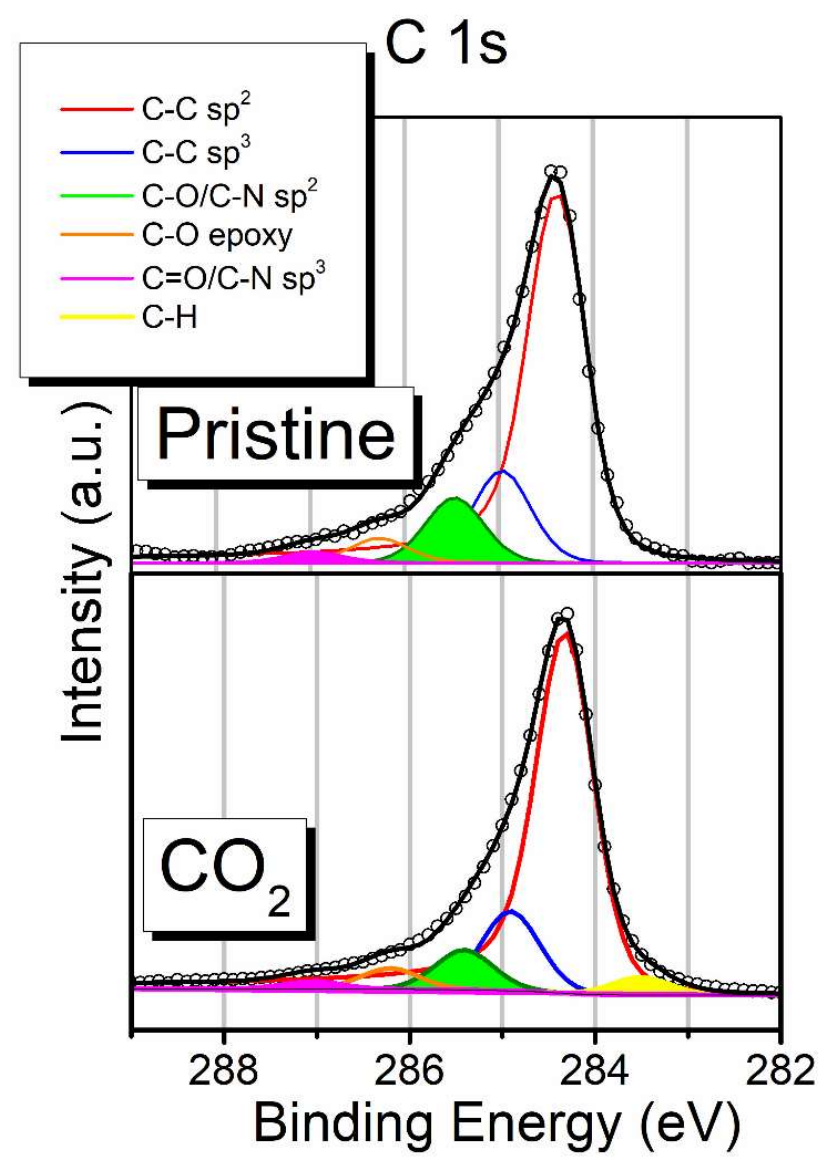

Figure S1. High Resolution C 1s region of an XPS spectrum obtained from a pristine sample and from the same sample following $\mathrm{CO}_{2}$ annealing. a.u. stands for arbitrary units. 


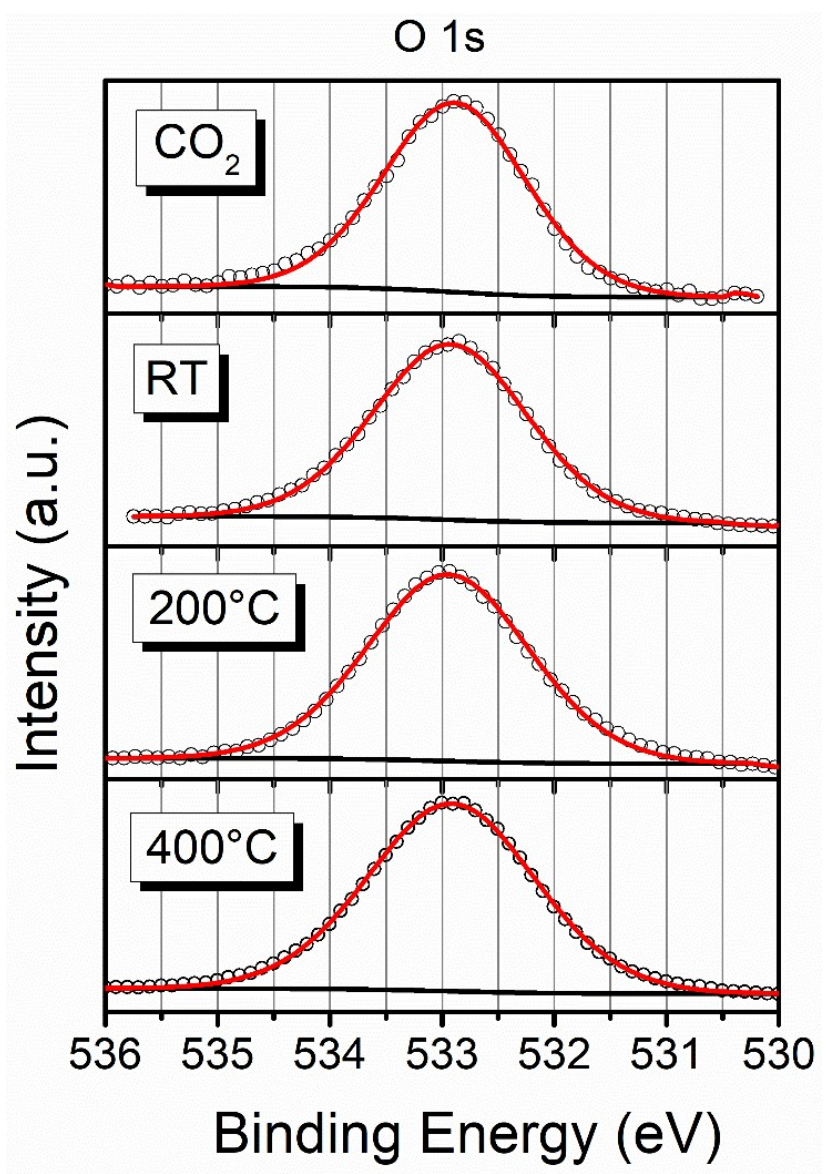

Figure S2. High Resolution O 1s region of XPS spectra obtained from samples following i) annealing at $500^{\circ} \mathrm{C}$ in $\mathrm{CO}_{2}$, ii) the same as in i) following annealing in ${ }^{14} \mathrm{NO}$ at $\mathrm{RT}, 200$ and $400^{\circ} \mathrm{C}$. a.u. stands for arbitrary units.

The use of this isotopically enriched gas enabled the use of NRA to quantify nitrogen incorporated in graphene. Nevertheless, as shown in Fig. S3, there was no detectable signal of alpha particles, evidencing that ${ }^{15} \mathrm{~N}$ is incorporated in amounts below the sensitivity of the technique $\left(\sim 1 \times 10^{14}\right.$ atoms $\left./ \mathrm{cm}^{2}\right)$. 


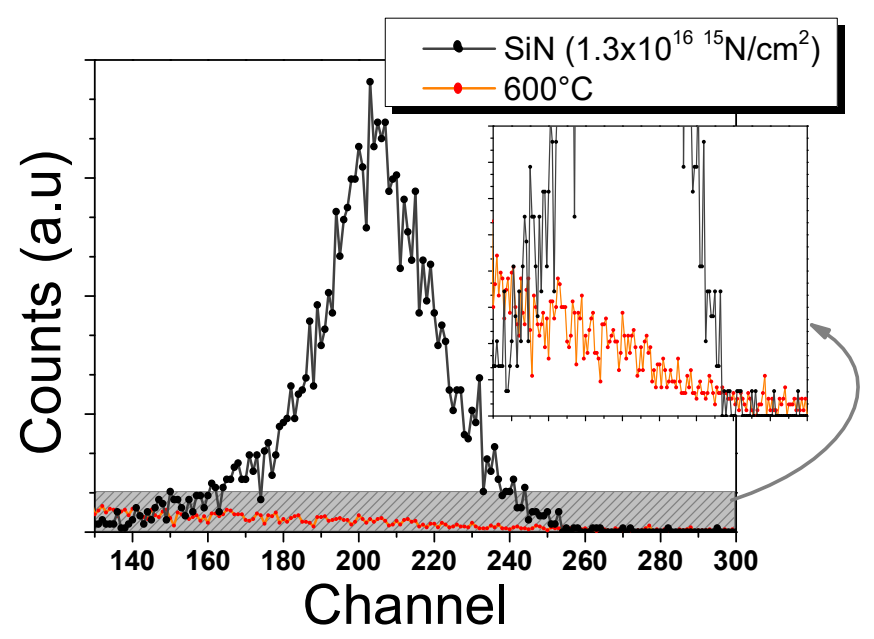

Figure S3 - Spectra of ${ }^{15} \mathrm{~N}(\mathrm{p}, \alpha){ }^{12} \mathrm{C}$ nuclear reaction performed with a proton beam of $950 \mathrm{keV}$ for a silicon nitride standard (black circle) and for a sample treated in 300 mbar of ${ }^{15} \mathrm{NO}$ at $600^{\circ} \mathrm{C}$ (red circle, $\mathrm{CO}_{2}$ pre-annealing was also performed).

Additional modifications in Raman spectra of samples were observed. In Fig. S4, the position of the G peak for annealed samples are shown. This parameter is related to doping of the graphene layer. A blue-shift of this peak is an indication of p-type doping. 


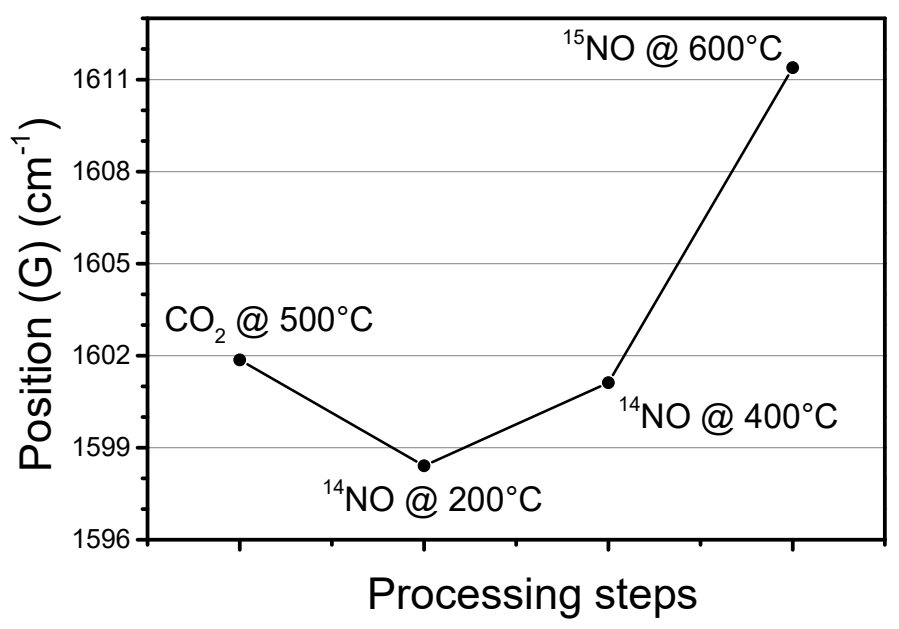

Figure S4. Position of the G peak in Raman spectra obtained from samples following i) annealing at $500^{\circ} \mathrm{C}$ in $\mathrm{CO}_{2}$, ii) the same as in i) following annealing in ${ }^{14} \mathrm{NO}$ at 200 and $400^{\circ} \mathrm{C}$, and iii) the same as in i) following annealing in ${ }^{15} \mathrm{NO}$ (purified gas) at $600^{\circ} \mathrm{C}$.

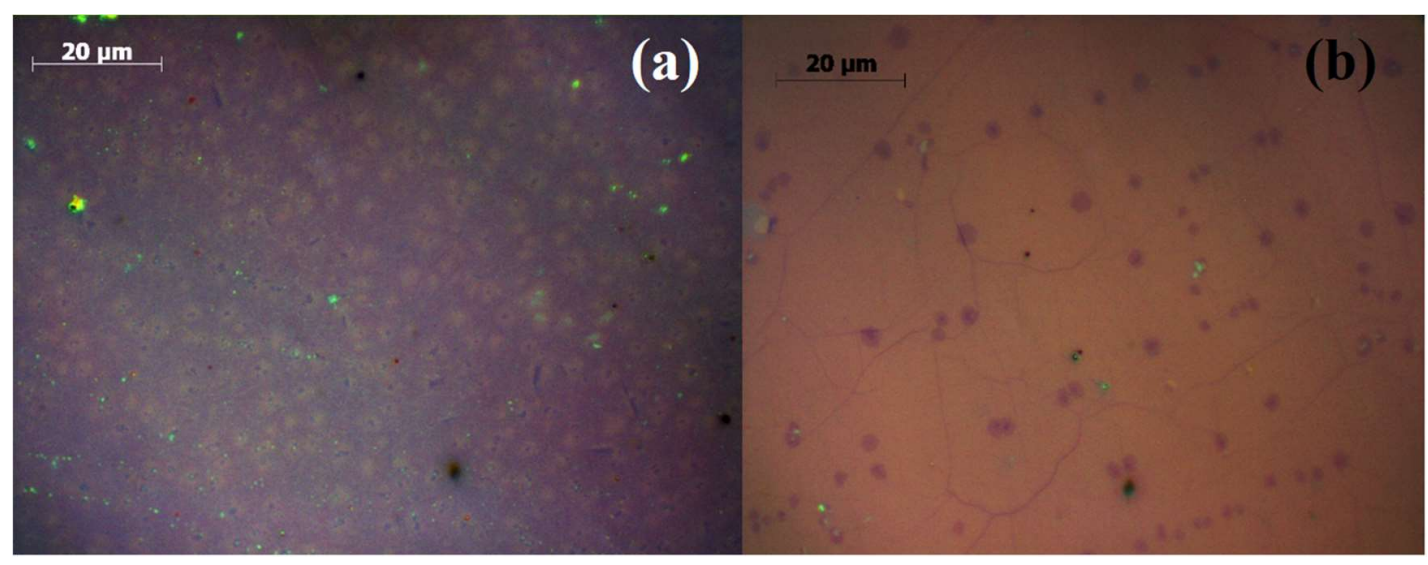

Figure S5 - Optical microscopy images of (a) a graphene/ $\mathrm{SiO}_{2} / \mathrm{Si}$ sample treated in $300 \mathrm{mbar}$ of $\mathrm{NO}$ at $400^{\circ} \mathrm{C}$ and (b) a graphene $/ \mathrm{SiO}_{2} / \mathrm{Si}$ sample treated in $300 \mathrm{mbar}$ of $\mathrm{NO}$ at $200^{\circ} \mathrm{C}\left(\mathrm{CO}_{2}\right.$ preannealing was also performed in both samples). 


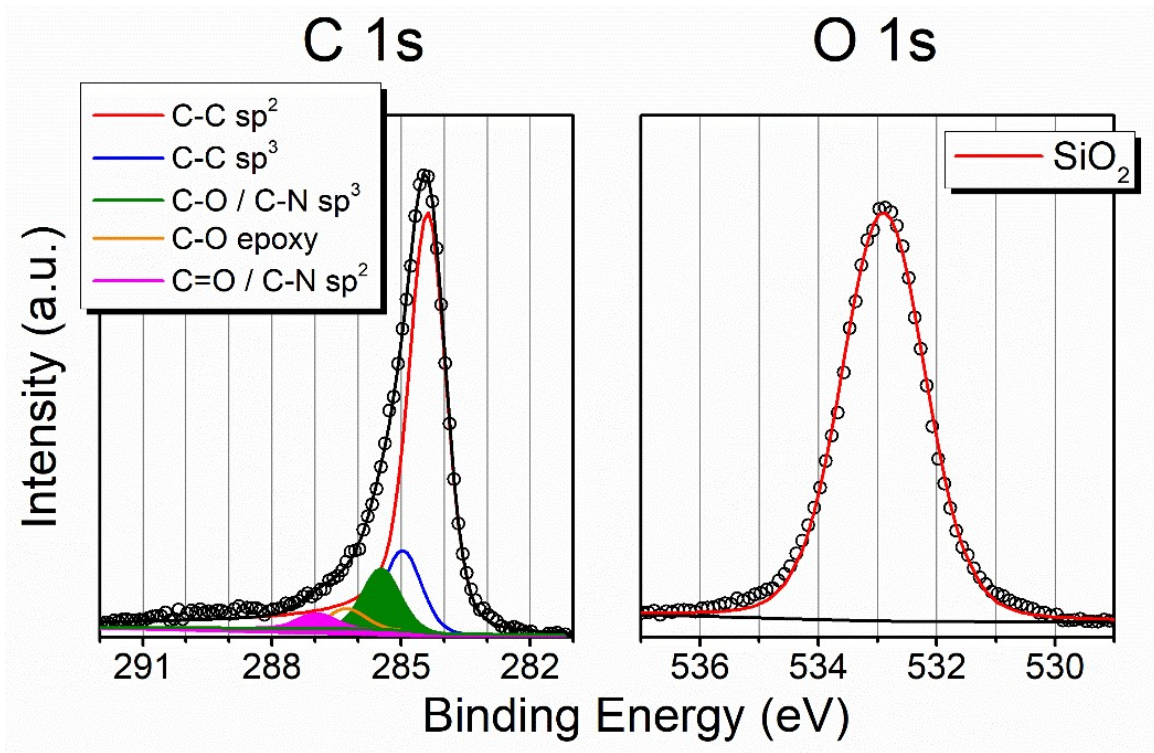

Figure S6. C 1s and O 1s regions of XPS spectra obtained from the sample last annealed at $600^{\circ} \mathrm{C}$ in purified ${ }^{15} \mathrm{NO}$. a.u. stands for arbitrary units. These spectra were obtained with a conventional $\mathrm{Al}$ anode X-ray source.

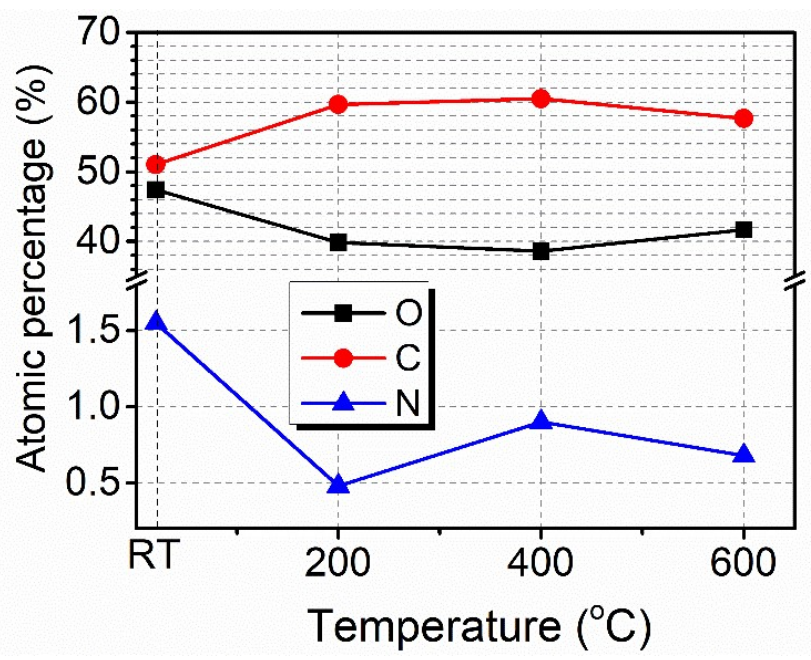

Figure S7. N, C, and O atomic percentages determined from XPS spectra of samples last annealed in ${ }^{15} \mathrm{NO}$ at the indicated temperatures. RT and a.u. stand for room temperature and arbitrary units, respectively. These spectra were obtained with a conventional $\mathrm{Al}$ anode $\mathrm{X}$-ray source. 